University of Nebraska - Lincoln

DigitalCommons@University of Nebraska - Lincoln

November 1988

\title{
Structure and magnetic properties of nanostructured Dy/ transition-metal multilayered films
}

\author{
Z.S. Shan \\ University of Nebraska - Lincoln \\ David J. Sellmyer \\ University of Nebraska-Lincoln, dsellmyer@unl.edu
}

Follow this and additional works at: https://digitalcommons.unl.edu/physicssellmyer

Part of the Physics Commons

Shan, Z.S. and Sellmyer, David J., "Structure and magnetic properties of nanostructured Dy/transitionmetal multilayered films" (1988). David Sellmyer Publications. 130.

https://digitalcommons.unl.edu/physicssellmyer/130

This Article is brought to you for free and open access by the Research Papers in Physics and Astronomy at DigitalCommons@University of Nebraska - Lincoln. It has been accepted for inclusion in David Sellmyer Publications by an authorized administrator of DigitalCommons@University of Nebraska - Lincoln. 


\title{
Structure and magnetic properties of nanostructured Dy/transition-metal multilayered films
}

\author{
Z.S. Shan and D. 3. Sellmyer \\ Behlen Laboratory of Physics, University of Nebraska, Lincoin, Nebraska 68588-0111
}

\begin{abstract}
We report the results of magnetic and microstructural studies for $\mathrm{T} / \mathrm{Dy}(\mathrm{T}=\mathrm{Fe}, \mathrm{Co}, \mathrm{Ni})$ compositionaliy modulated films prepared in a multiple-gun sputtering system. The perperdicular anisotropy and magnetization were measured systematically for $\not-\AA$ Fe/ $Y$ - $\AA$ Dy and $X-\AA \mathrm{Co} / Y-\AA$ Dy films. The layer-thickness dependence of the magnetization for $\mathrm{Co} /$ Dy and Fe/Dy was interpreted in terms of the antiparalle coupling between transition-metal and Dy magnetic moments. For $\mathrm{C} O / D y$ films the ranges of $X$ and $Y$ required for perpendicular anisotropy were determined. A comparision of the structural and magnetic properties of $\mathrm{Ni} /$ Dy, Co/Dy, and Fe/Dy is given and the origin of the perpendicular anisotropy is discussed.
\end{abstract}

\section{MTRODUGTION}

Compositionally modulated films have been studied extensively for both physical and technological reasons. Some films ${ }^{t-3}$ are of great interest for their strong perpendicular anisotropy. They could be the materials of choice for investigating solid interfaces or candidates for perpendicular recording. Previously we have studied $\mathrm{Fe} / \mathrm{Ta}, \mathrm{Fe} / \mathrm{Dy}$, and $\mathrm{Fe} /$ Nd multilayered fims, ${ }^{4-6}$ and the present work represents an extension to $\mathrm{Co} / \mathrm{Dy}, \mathrm{Fe} / \mathrm{Dy}$, and $\mathrm{Ni} / \mathrm{Dy}$ systems. The layerthickness dependence of the magnetization and perpendicular anisotropy was investigated systematically. In this paper we shall only report some of the magnetic properties at room temperature. A more detalled discussion of the temperature dependence of the magnetic properties and the origin of the perpendicular anisotropy will be published elsewhere.

\section{EXPERIMENT}

We have prepared a variety of sampies of the form $X-\AA \mathrm{T} / Y \cdot \AA \mathrm{Dy}(\mathrm{T}=\mathrm{Fe}, \mathrm{Co}$, and $\mathrm{N})$. Here $X$ and $Y$ are the layer thicknesses of the transition metal and $D y$. In this work $X$ and $Y$ range from 2.5 to $160 \AA$ and 1.75 to $224 \AA$, respectively. In order to study the layer-thickness dependence of magnetic properties systematically, in the region of $2.5 \AA$ $<X<20 \AA$ and $3.5 \AA<Y<14 \AA$ the interval for $X$ and $Y$ is about one atomic diameter, i.e., $2.5 \AA$ for $T$ and $3.5 \AA$ for $D y$. In some regions the interval is only $1.25 \AA$ to probe the change of the magnetic properties more precisely. Totally about 150 samples have been prepared.

The samples were prepared with the multiple-gun sputtering system. The preparation conditions here are the same as those mentioned in Ref. 6 . In this system six samples can be made in one vacuum run by means of a specially designed mechanism which can be rotated to move the substrate shutter from one position to another. This design enables the sample preparation to be done much more efficiently and reduces the fuctuation of magnetic properties due to the change of preparation conditions among different runs.

The measurements of the magnetic properties, such as the magnetization in the direction perpendicular and parallel to the film plane, coercivity, and anisotropy energy, etc., were performed with vibrating sample magnetometry. The atomic structure was studied with large and small-angle $\mathrm{x}$ ray diffraction.

\section{RESUETS ANO DISCUSSIOA}

Four examples of large-angle $x$-ray diffraction patterns are shown in Fig. 1, and three examples of small-angle $x$-ray diffraction in Fig. 2 . We notice that $40-\AA$ Fe/56- $\AA$ Dy film has much sharper diffraction peaks than those of the $40-\AA$ $\mathrm{Co} / 56-\AA \mathrm{Dy}$ film. Therefore, in rough terms, $40-\AA \mathrm{Fe} / 56-\AA$ Dy has crystalline structure and $40-\AA \mathrm{Co} / 56-\AA$ Dy has microcrystalline structure, whereas for $15-\AA \mathrm{Fe} / 14-\mathrm{A} D \mathrm{Dy}$ and 10- $\AA \mathrm{Co} / 14-\AA$ Dy fims, the structure is amorphous. The small-angle $x$-ray diffraction patterns only show the firstorder peaks for $40-\AA \mathrm{Fe} / 14-\AA \mathrm{A}$ Dy and $50-\AA \mathrm{Co} / 14-\AA \mathrm{Dy}$, but also the second-order peak for $30-\AA \mathrm{Ni} / 7-\hat{\AA}$ Dy. This implies that the compositionally modulated structure has the sinusoidal form for $40-\AA \mathrm{Fe} / 14-\AA$ Dy and $50-\AA \mathrm{Co} / 14-$ $\hat{\AA} \mathrm{Dy}$, and a sharper interface boundary for $30-\hat{\AA} \mathrm{Ni} / 7-\AA$ Dy.

The layer-thickness dependence of magnetic properties was probed in terms of the measurements of hysteresis loops for all samples. One series of samples for $\mathrm{Co} / \mathrm{Dy}$ is shown in Fig. 3. In this figure Dy layer thickness is fixed at 5 and the Colayer thickness changes from 3.5 to $10 \AA$. It is seen clearly that: (i) The samples with $X=3.5,6$, and $8 \AA$ have strong

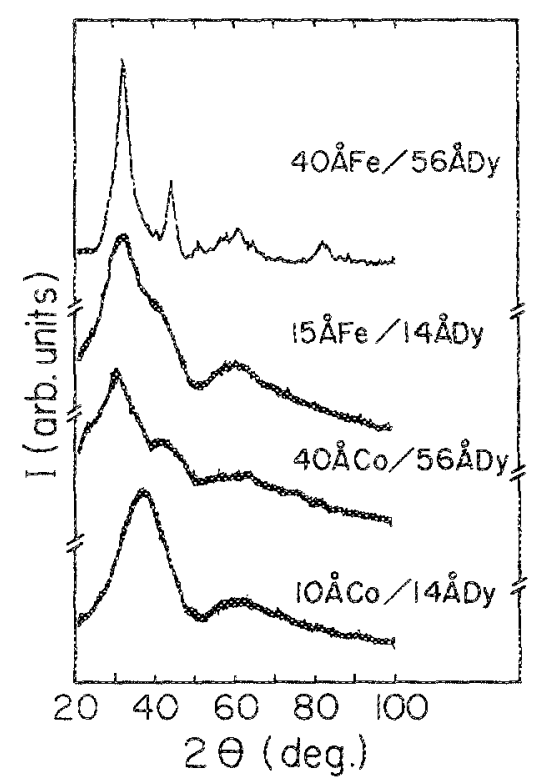

FIG. 1. CuKa large-angle diffraction intensity as a function of $2 \theta$ for four $\mathrm{Fe} /$ Dy and Co/Dy samples. 


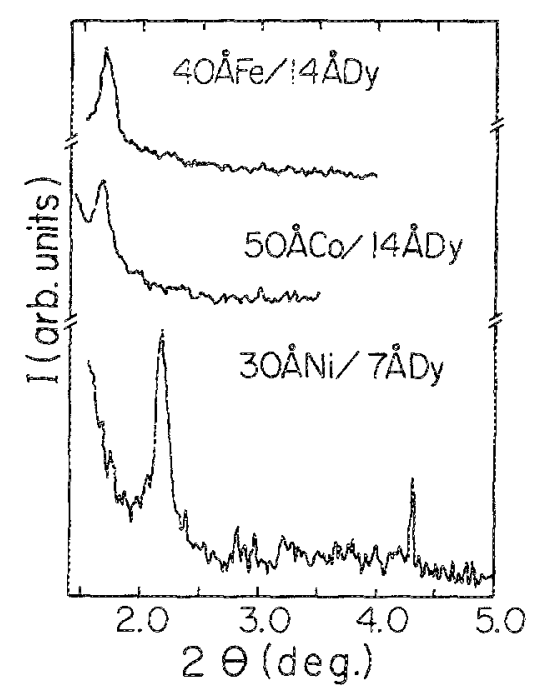

FIG. 2. CuK $\alpha$ small-angle diffraction intensity as a function of $2 \theta$ for three $\mathrm{ge} /$ Dy, Co/Dy, and Ni/Dy samples.

perpendicular anisotropy, i.e., $\sigma_{1}>\sigma_{1 j}$. But as the Co layer thickness reaches $10 \AA$, $\sigma_{\|}$becomes larger than $\sigma_{1}$. (ii) The values of covercivity $H_{c}$ and magnetization $\sigma$ ( $8 \mathrm{kOe}$ ) shown on the figure are changed correspondingly as the $\mathrm{Co}$ layer thickness increases, and this will be discussed in more detail in the following. (iii) The sample of $6-\AA \mathrm{Co} / 5-\AA \mathrm{Dy}$ has interesting character for its "square hysteresis loop," larger coercivity of $2.6 \mathrm{kOe}$, and stronger perpendicular anisotropy. The fact that the samples with thinner Co layer have stronger perpendicular anisotropy means the interfaces play an important role for perpendicular anisotropy. The interface possesses reduced symmetry which favors perpendicular anisotropy as indicated in Ref. 7.

Based on systematic measurements, the layer-thickness dependence of the magnetization $\sigma_{1}$ at $8 \mathrm{kOe}$ for $\mathrm{Co} / \mathrm{Dy}$ films is summarized in Fig. 4 . The effect of the antiferromagnetic coupling between $C o$ and Dy magnetic moments is shown clearly in this figure: (i) For example, the magnetization of $X-\AA \mathrm{A} C \mathrm{C} / 8-\AA \mathrm{D}$ y is saturated for thick Co layer be-

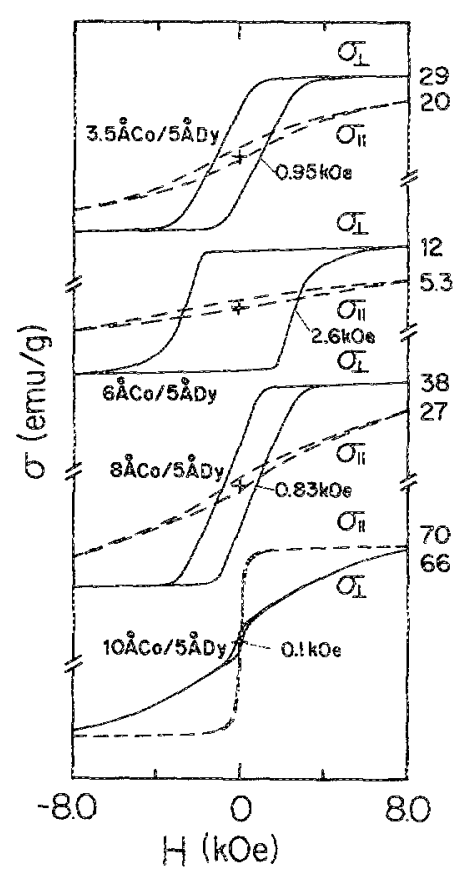

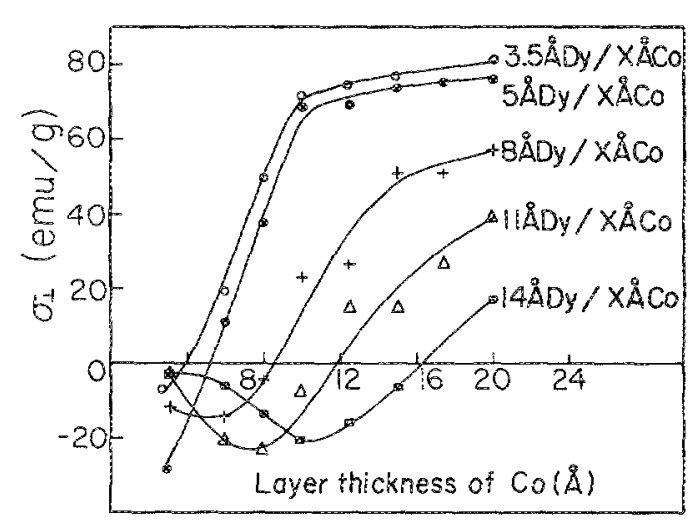

FIG. 4. Layer-thickness dependence of magnetization $\sigma_{1}$ at room temperature.

cause of the Co magnetization dominance in this region. As the Co layer becomes thinner $\sigma_{l}$ decreases, passes through the compensation point, and changes the sign since the Dy magnetization dominates. At very small Co thickness $\left|\sigma_{1}\right|$ decreases, presumably because both the atomic and magnetic structures become highly disordered. (ii) As the Dy layer gets thicker the compensation point moves towards larger Co layer thickness, which is reasonable.

It is useful to determine the ranges of Co and Dy layer thickness exhibiting perpendicular anisotropy. The result is shown in Fig. 5. The rather broad region exhibiting perpendicular anisotropy suggests that Co/Dy films could be promising for perpendicular recording.

It is of interest to ask whether the thickness dependence of the magnetic properties discussed above could be explained entirely on the basis of a model in which one assumes a composition dependence of an essentially homogeneous glassy alloy. Figure 6 gives a negative answer to this question. The hysteresis loops of four samples, which have identical chemical ratios of $C o$ and $D y$, change their shape regulari $y$ as the nominal layer thickness of Co and $D y$ increase. This feature means the compositionally modulated structure clearly exists and controls the magnetic properties.

A similar character of the layer-thickness dependence of magnetic properties, as shown in Figs. 3-5, is also found for Fe/Dy fims. Some of these figures were shown in our previous paper.

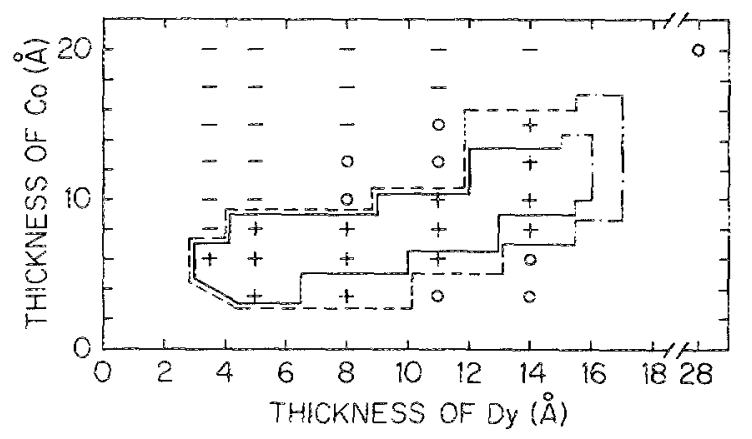

FIG. 5. The range of layer thickness exhibiting perpendicular or parallel anisotropy characteristics for Co/Dy films. $+=\sigma_{1}>\sigma_{\|} ; O=\sigma_{L} \approx \sigma_{\|}$; and $\cdots=\sigma_{1}>\sigma_{1}$. The region surrounded by the solid line possesses $\left(\sigma_{1} / \sigma_{1}\right)>2$ at $H=0$ 


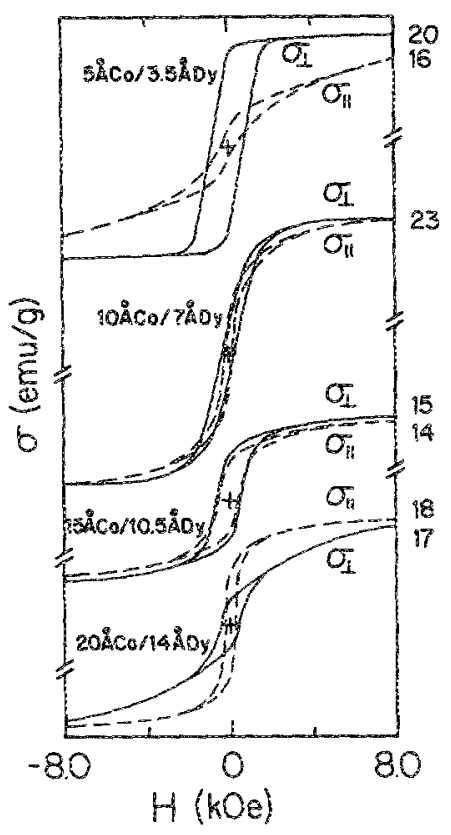

Two series of Ni/Dy samples, the $X-\AA \mathrm{Ni} / 3.5-\AA$ Dy and $X-\AA \mathrm{Ni} / 7-\AA \operatorname{Dy}(X=2.5,5,7.5,10,15,20$, and $30 \AA)$, have been studied. The magnetizations at room temperature are much weaker than those of $F$ e/Dy and $C o / D y$ films. This follows from the weaker exchange coupling in $\mathrm{Ni}$ as conpared to Fe and $\mathrm{Co}$. Low-temperature studies of the $\mathrm{Ni} / \mathrm{Dy}$ system are now under way.

The magnetic structure and origin of the perpendicular anisotropy for the compositionally modulated films are in:teresting but complicated. Many models, such as stress induced anisotropy, ${ }^{8}$ single-ion anisotropy, ${ }^{9}$ exchange anisotropy ${ }^{30}$ anisotropic ordering of atomic pairs, ${ }^{3}$ etc., have been proposed to interpret the perpendicular anisotropy. In this paper we have pointed out that the interface is the main source for the perpendicular anisotropy, but a microscopic model sutable for the compositionally modulated films remains to be developed. For the T/Dy ( $T=F e, C o$, and $\mathrm{Ni}$ ) films the following conclusions can be stated: (i) Stress induced anisotropy is not important based on our experiment that the $5-\mathrm{A}$. $/ 5-\AA$ Dy film deposited on Mylar, $\mathrm{Ta}, \mathrm{Cu}$, and mica substrates exhibited almost same magnetic properties (not shown in this paper). (ii) The model of anisotropic ordering of the atomic pairs is appealing for explaining the perpendicular anisotropy, as is qualitatively discussed in Ref. 3. (iii) The single-ion model is successful in calculating the perpendicular anisotropy for nonmultilayered $\left(\mathrm{Gd}_{0.75} \mathrm{R}_{0.25}\right)_{19} \mathrm{CO}_{81} .{ }^{9}$ This may be a promising model, but the anisotropic distribution of the atoms in the interface must be determined carefuily. In particular, the interactions of the rare earth with both its rare-earth and transition-metal neighbors must be accounted for properly.

In summary, the systematic studies of the layer-thickness dependerce of the magnetization and perpendicular anisotropy have been performed for $\mathrm{Co} / \mathrm{Dy}$ and $\mathrm{Fe} / \mathrm{Dy}$ compositionally modulated films. The values of magnetization and perpendicular anisotropy are controllable. Thus, these fims may be the potertial candidates for perpendicular and or magneto-optic recording. The origins of the perpendicuhar anisotropy have been discussed and a detailed model suitable for the compositionally modulated films presently is being investigated.

\section{ACKAOWLEOMENTS}

We are grateful for financial support to the Solid State Physics Program of NSF under Grant No. DMR-8605367. For assistance and helpful discussion we thank R. D. Kirby and $\mathbf{K}$. D. Aylesworth.

IF. I. A. den Broeder and H. C. Donkersloot, J. Appl. Phys. 61, 4317 (1987); P. F. Carcia, A. D. Meinhaldt, and A. Suna, Appl. Phys. Lett. 47, $178(1987)$.

${ }^{2}$ N. C. Koon, B. T. Jonker, F. A. Volkening, J. J. Krebs, and G. A. Prinz, Phys. Rev. Lett. 59, 2463 (1987).

${ }^{3}$ N. Sato and K. Habu, J. Appl. Phys. 61, 4287 (1987); N. Sato, K. Habu, and T. Oyama, IEEE Trans. Magn. MAG $-23,2614$ (1987).

${ }^{4}$ D. J. Selmyer, Z. E. Zhao, Z. S. Shan, and S. Nafs, J. Appl. Phys. 61, 4323 (1987).

${ }^{5}$ Z. S. Shan, Z. R. Zhao, J. G. Zhao, and D. J. Selmyer, J. Appl. Phys. 61, $4320(1987)$.

6Z. S. Shan, S. Nafis, K. D. Aylesworth, and D. J. Sellmyer, J. Appl. Phys. 63, 3218 (1988).

M. L. Neel, J. Phys. Rad. 15, 225 (1954)

${ }^{8} \mathrm{R}$. Zwingman, W. L. Wilson, Jr, and K. C. Bourne, Ir., AIP Conf. Proc. $34,334(1976)$.

${ }^{Y}$ Y. Suzuki, S. Takayama, F. Kirino, and N. Ohta, IEEE Trans. Magn. MAG-23, 2275 (1987).

1) W. H. Meiklejohn, F. E. Luborsky, and P. C. Frishchman, IEEE Trans. Magn. MAG-23, 2272 (1987). 